\title{
MULTIPLICATION OPERATORS ON VECTOR-VALUED FUNCTION SPACES
}

\author{
HÜLYA DURU, ARKADY KITOVER, AND MEHMET ORHON
}

(Communicated by Thomas Schlumprecht)

\begin{abstract}
Let $E$ be a Banach function space on a probability measure space $(\Omega, \Sigma, \mu)$. Let $X$ be a Banach space and $E(X)$ be the associated Köthe-Bochner space. An operator on $E(X)$ is called a multiplication operator if it is given by multiplication by a function in $L^{\infty}(\mu)$. In the main result of this paper, we show that an operator $T$ on $E(X)$ is a multiplication operator if and only if $T$ commutes with $L^{\infty}(\mu)$ and leaves invariant the cyclic subspaces generated by the constant vector-valued functions in $E(X)$. As a corollary we show that this is equivalent to $T$ satisfying a functional equation considered by Calabuig, Rodríguez, and Sánchez-Pérez.
\end{abstract}

\section{INTRODUCTION}

Our paper is motivated by the following question which continues to attract considerable attention. Let $M$ be a linear space of (scalar or vector-valued) functions on some set, let an algebra of scalar functions on the same set be given, and let $T: M \rightarrow M$ be a linear operator. What properties of $T$ guarantee that it is an operator of multiplication by some function in the given algebra of scalar functions? The answer often can be expressed in terms of $T$-invariant linear subspaces of $M$. On the other hand, it is well known (see [3]) that a linear operator on a vector lattice can be represented as a multiplication operator if and only if it is regular and band preserving. If the algebra of such operators is rich enough (e.g., if $M$ is a Banach lattice with a quasi-interior point), then we have another criterion: a regular operator is a multiplication operator if and only if it commutes with all regular band preserving operators. The first approach works for Banach $C(K)$ modules as well. If $M$ is a Banach $C(K)$-module, then a (bounded linear) operator $T$ on $M$ is induced by multiplication by a function from the closure of $C(K)$ in the weak-operator topology on $\mathcal{L}(M)$ if and only if $T$ leaves invariant every cyclic subspace $M(x)$ where $x \in M$ and $M(x)=\operatorname{cl}\{a x: a \in C(K)\}$ ([2, Theorem 6.2], 6, Theorem 7]). But this condition is quite strong and not so easy to verify. Inspired by the paper of Calabuig, Rodríguez, and Sánchez-Pérez [4, we looked at the case when the operator leaves invariant only some special cyclic subspaces. More precisely, we look at the case when the Banach $C(K)$-module (with $C(K)=L^{\infty}(\mu)$ ) can be represented as a Banach space of vector-valued measurable functions and

Received by the editors April 5, 2011 and, in revised form, December 15, 2011.

2010 Mathematics Subject Classification. Primary 47B38; Secondary 46G10, 46B42, 46H25.

Key words and phrases. Multiplication operator, Köthe-Bochner space, vector-valued measurable function, Banach function space, Banach lattice, ideal center, Banach $C(K)$-module.

The first author was supported by the Scientific Projects Coordination Unit of Istanbul University, project No. 3952 . 
the special cyclic subspaces are generated by the constant vector-valued functions. The condition that an operator leaves such subspaces invariant is in general not sufficient for being a multiplication operator, and we have to additionally require that the restriction of the operator on such a subspace commutes with the restrictions of multiplication operators to the same subspace (or more strongly, that the operator commutes with the multiplication operators everywhere). The techniques developed in the paper allowed us to show that these two conditions together already become sufficient to identify multiplication operators on a very broad class of spaces of vector-valued measurable functions. Thus we considerably extend the results in [4].

\section{SPACES OF VECTOR-VAlued CONTINUOUS FunCtions}

Let $K$ be a compact Hausdorff space and let $C(K)$ denote the algebra of realvalued continuous functions on $K$. Given a real Banach space $X$, by $C(K, X)$ we denote the Banach space of continuous $X$-valued functions on $K$. For each $f \in$ $C(K, X)$ by $|f|_{X} \in C(K)$ we denote its norm function. That is,

$$
|f|_{X}(t)=\|f(t)\|
$$

for each $t \in K$. Furthermore $\|f\|=\left\||f|_{X}\right\|_{C(K)}$ gives the norm on the space $C(K, X)$. For each $a \in C(K)$, one defines the product af $\in C(K, X)$ pointwise in the usual manner. With this product, $C(K, X)$ is a Banach $C(K)$-module. For each $x \in X$, we denote by $\vec{x} \in C(K, X)$ the constant function defined by $\vec{x}(t)=x$ for each $t \in K$. Let $M(X)$ denote the submodule of $C(K, X)$ generated by the constant functions. When $f \in C(K, X)$, its range $f(K)$ is compact. Therefore $f(K)$ is a separable subset of $X$. On the other hand, it is clear that the range of each function in $M(X)$ is contained in a finite dimensional subspace of $X$. Moreover, if $f \in C(K, X)$ has its range contained in a finite dimensional subspace of $X$, then $f$ is in $M(X)$. That is, $f=\sum a_{i} \vec{x}_{i}$ for $a_{i} \in C(K)$ and $x_{i} \in X$ with $i=1,2, \ldots, n$ for some positive integer $n$.

Lemma 2.1. $M(X)$ is dense in $C(K, X)$.

Proof. Let $f \in C(K, X)$ and $\varepsilon>0$. There exist $\left\{x_{1}, x_{2}, \ldots, x_{n}\right\}$ in $f(K)$ such that the open balls $B\left(x_{i}, \varepsilon\right)$ in $X$ with $i=1,2, \ldots, n$ cover $f(K)$. Let $U_{i}=f^{-1}\left(B\left(x_{i}, \varepsilon\right)\right)$ and $t_{i} \in U_{i}$ such that $f\left(t_{i}\right)=x_{i}$ with $i=1,2, \ldots, n$. The collection $\left\{U_{i}: i=\right.$ $1,2, \ldots, n\}$ is a finite open cover of $K$. Let the collection $\left\{a_{i}: i=1,2, \ldots, n\right\}$ in the positive unit ball of $C(K)$ be a partition of unity subordinate to the cover $\left\{U_{i}: i=1,2, \ldots, n\right\}$. Let $g=\sum a_{i} \vec{x}_{i}$. Then $\|f-g\|<\varepsilon$.

Let $F$ be a Banach lattice with a quasi-interior point $u$ [13, II.6.1]. That is, $u$ is a non-negative element of $F$ such that the closed ideal $I(u)$ generated by $u$ is $F$. Note that a subspace $I$ of $F$ is called an (order) ideal if $x \in I, y \in F$ and $|y| \leq|x|$ implies that $y \in I$. Recall that an operator $T$ on $F$ is in the ideal center if and only if for some $\lambda>0,|T(x)| \leq \lambda x$ for each non-negative $x \in F$. The ideal center of $F$, denoted by $Z(F)$, is a Banach lattice and a commutative Banach algebra. In fact $Z(F)$ is represented isometrically as $C(K)$ for some compact Hausdorff space $K$, both as an algebra and as a Banach lattice [1, Theorems 3.31, 3.34]. That $F$ has a quasi-interior point $u$ means that $Z(F) u$ is dense in $F$ [15, Proposition 1.1]. (Because of this fact a quasi-interior point is also called a topological order unit.) Then for each $x \in F$, the closure of $Z(F) x$ is equal to the closed ideal $I(x)$ of $F$ 
generated by $x$ [15]. Without loss of generality we suppose $\|u\|_{F}=1$. Then we have that $F$ is a Banach $C(K)$-module. Also it is well known [13, Theorem III.4.5] that $F$ has a functional representation as an ideal in $C_{\infty}(K)$, the set of extended continuous functions on $K$ into $[-\infty, \infty]$. Recall that a continuous function on $K$ into $[-\infty, \infty]$ is called an extended continuous function if it is finite on a dense open subset of $K$. For each $a \in C(K)$, au in $F$ is represented by $a$ in $C_{\infty}(K)$. In particular $u$ is represented by 1 and $C(K)$ in $C_{\infty}(K)$ corresponds to the ideal generated by $u$ in $F$. In view of this we will always think of $F$ in terms of its representation in $C_{\infty}(K)$ and replace the quasi-interior point $u$ by the function 1.

We introduce a new norm on $C(K, X)$ by

$$
\|f\|_{F(X)}=\left\||f|_{X}\right\|_{F}
$$

for each $f \in C(K, X)$. Let $F_{\pi}(X)$ denote the completion of $C(K, X)$ in this norm. It is clear that $F_{\pi}(X)$ is also a Banach $C(K)$-module. Let $f \in F_{\pi}(X) \backslash C(K, X)$ and let $\left\{f_{n}\right\}$ be a sequence in $C(K, X)$ that converges to $f$ in $F_{\pi}(X)$. Then the sequence of norm functions $\left\{\left|f_{n}\right|_{X}\right\}$ is a Cauchy sequence in $F$ and converges to a non-negative element of $F$. We will denote the limit point by $|f|_{X}$ and call it the norm function of $f \in F_{\pi}(X)$. We are justified in this since $\|f\|_{F(X)}=\left\||f|_{X}\right\|_{F}$ and $|f|_{X} \in C_{\infty}(K)$.

It is well known (see, e.g., [14, 13, Chapter V.3], 7]) that each cyclic subspace of a Banach $C(K)$-module $M$ may be represented as a Banach lattice with quasiinterior point. Namely, for each $x \in M$, the cyclic subspace $M(x)=\operatorname{cl}(C(K) x)$ is a Banach lattice with positive cone $\operatorname{cl}\left(C(K)_{+} x\right)$ and quasi-interior point $x$. In general, the ideal center of $M(x)$ is $w$ - $c l\left(C(K)_{\mid M(x)}\right)$ (see, e.g., [11, Theorem 1]). Here ' $c l$ ' denotes closure in norm in $M$ and ' $w$ - $c l$ ' the closure in the weak operator topology when we consider the representation of $C(K)$ as an algebra of operators on $M(x)$. In the case of $F_{\pi}(X)$, we get more precise information on the structure of its cyclic subspaces.

Lemma 2.2. For each $f \in F_{\pi}(X)$, the cyclic subspace $F(f)$ is isometric and lattice isomorphic to the closed ideal $I\left(|f|_{X}\right)$ of $F$. In particular, for each $0 \neq x \in X$, the cyclic subspace $F(\vec{x})$ is isometric and lattice isomorphic to $F$.

Proof. Let $f \in C(K, X)$ and $a \in C(K)$. Then, for each $t \in K$,

$$
|a f|_{X}(t)=\|a(t) f(t)\|=|a(t)|\|f(t)\|=|a(t)||f|_{X}(t) .
$$

Therefore $|a f|_{X}=|a||f|_{X}$. It follows that

$$
\|a f\|_{F(X)}=\left\||a||f|_{X}\right\|_{F}=\left\|a|f|_{X}\right\|_{F} .
$$

Then the same equality also follows for $f \in F_{\pi}(X) \backslash C(K, X)$ for any $a \in C(K)$. This proves that the submodule generated by $f$ in $F_{\pi}(X)$ is isometric and lattice isomorphic to the sublattice $C(K)|f|_{X}$ of $F$. By passing to the closure we complete the first part of the result. For any $x \in X$, we have $|\vec{x}|_{X}=\|x\| 1$ in $F$. Since 1 is a quasi-interior point, we have $F(\vec{x}) \cong F$.

We will call an operator $T$ on a Banach $C(K)$-module $M$ a multiplication operator if $T(x)=a x$ for some $a \in C(K)$, for all $x \in M$. For a Banach lattice $F$ with quasi-interior point and ideal center $Z(F)=C(K)$, the ideal center is maximal abelian (see, e.g., [15, Theorem 2.4], [11, Corollary 3]). That is, an operator on $F$ which commutes with $C(K)$ is a multiplication operator. 
Theorem 2.3. Suppose $K$ is a compact Hausdorff space and $F$ is a Banach lattice with quasi-interior point $u$ and ideal center $Z(F)=C(K)$. Suppose $X$ is a Banach space. Let $T$ be an operator on $F_{\pi}(X)$. Consider the following conditions:

(i) $T$ is a multiplication operator.

(iia) For each $x \in X$, the cyclic subspace $F(\vec{x})$ is left invariant by $T$.

(iib) For each $x \in X, T$ commutes with $C(K)$ on $F(\vec{x})$.

Then (i) $\Leftrightarrow$ (iia) and (iib).

Proof. The implication (i) $\Rightarrow$ (iia) and (iib) is clear. Conversely, assume that (iia) and (iib) hold. Let $x \in X$. From Lemma 2.2, we have that $F(\vec{x})$ is isometric and lattice isomorphic to $F$. Then (iia) implies that we may consider $T$ restricted to $F(\vec{x})$ as an operator on $F$. Then (iib) and the discussion preceding the theorem imply that $T$ is a multiplication operator on $F(\vec{x})$. That is, there exists $a_{x} \in C(K)$ such that $T(f)=a_{x} f$ for all $f \in F(\vec{x})$. Suppose $x, y \in X$ are linearly independent. Then

Therefore

$$
a_{x} \vec{x}+a_{y} \vec{y}=T(\vec{x}+\vec{y})=a_{x+y}(\vec{x}+\vec{y})
$$

$$
\left(a_{x}-a_{x+y}\right) \vec{x}=\left(a_{x+y}-a_{y}\right) \vec{y} .
$$

From the linear independence of $x, y$, it easily follows that

$$
\left(a_{x}-a_{x+y}\right)=\left(a_{x+y}-a_{y}\right)=0 .
$$

Hence there exists an $a \in C(K)$ such that $T(f)=$ af for all $f \in M(X)$. By Lemma 2.1, $M(X)$ is dense in $C(K, X)$, and by construction $C(K, X)$ is dense in $F_{\pi}(X)$. This means that $M(X)$ is dense in $F_{\pi}(X)$. Therefore by continuity $T$ is multiplication by $a$ on all of $F_{\pi}(X)$.

We point out that Lemma 2.2 and Theorem 2.3 hold for $C(K, X)$. Namely let $F=C(K)$; then $F_{\pi}(X)=C(K, X)$.

\section{KÖTHE-BOCHNER SPACES}

Let $(\Omega, \Sigma, \mu)$ be a complete probability measure space. Let $L(\mu)$ denote the Dedekind complete vector lattice of the equivalence classes of almost everywhere finite measurable functions over $(\Omega, \Sigma, \mu)$. Recall that a vector lattice is called Dedekind complete if for each non-empty subset $A$ that is bounded above in the vector lattice, a least upper bound, $\sup A$, exists. The ideal center of $L(\mu)$ is $L^{\infty}(\mu)=C(S)$. Due to the fact that $L^{1}(\mu)^{*}=L^{\infty}(\mu)$, the space $S$ is hyperstonian. The characteristic functions of the sets of positive measure in $\Sigma$ correspond to the characteristic functions of the clopen subsets of $S$. This correspondence gives the identification between $L^{\infty}(\mu)$ and $C(S)$. We will also assume the identification of $L(\mu)$ with $C_{\infty}(S)$. We denote by $E$ a Banach function space in $L(\mu)$. We assume that $1 \in E$ with unit norm, that $E$ is an ideal in $L^{1}(\mu)$, and that this embedding is continuous. Clearly $E$ is Dedekind complete and 1 is a weak order unit in $E$ (i.e., $1 \wedge|\phi|=0$ for some $\phi \in E$ implies $\phi=0$ ). We let $F=I(1)$, the Banach function space given by the closed ideal generated by 1 in $E$. Then 1 is a quasiinterior point of $F$. $L^{\infty}(\mu)$ is the ideal center of both $E$ and $F$. Let $X$ be a Banach space. On $X$, we take the Borel $\sigma$-algebra with respect to the norm. By $L(\mu, X)$ we denote the $L^{\infty}(\mu)$-module consisting of the equivalence classes of the strongly measurable functions on $\Omega$ into $X$. That is, $f \in L(\mu, X)$ means $f: \Omega \rightarrow X$ is 
measurable and $f(\Omega \backslash A)$ is separable in $X$ for some $A \in \Sigma$ with $\mu(A)=0$. As in the previous section, $|f|_{X} \in L(\mu)$ will denote the norm function of a strongly measurable function $f$. The Köthe-Bochner space $E(X)$ is defined (see, e.g., 10, Section 3.4]) to consist of all functions $f \in L(\mu, X)$ with $|f|_{X} \in E$. It can be shown that $E(X)$ is a Banach $L^{\infty}(\mu)$-module when it is equipped with the norm

$$
\|f\|_{E(X)}=\left\||f|_{X}\right\|_{E} .
$$

When $E$ is $L^{1}(\mu)$ or $L^{\infty}(\mu)$, as usual, we denote $E(X)$ by $L^{1}(\mu, X)$ and $L^{\infty}(\mu, X)$ respectively. $L^{1}(\mu, X)$ is called the space of Bochner-integrable functions [5, II.2.2]. It is clear from the definition that we always have the continuous embeddings

$$
L^{\infty}(\mu, X) \subset E(X) \subset L^{1}(\mu, X) .
$$

We denote by $S(X)$ the functions with finite range and by $\sigma-S(X)$ the functions with countable range in $L(\mu, X)$. Let $\left\{e_{i}: i \in \mathcal{I}\right\}$ be a set of pairwise disjoint idempotents in $L^{\infty}(\mu)$ and let $\left\{x_{i}: i \in \mathcal{I}\right\}$ be a set of distinct elements in $X$ for some index set $\mathcal{I}$. If $\mathcal{I}$ is finite, $f=\sum e_{i} \vec{x}_{i}$ is in $S(X)$; and if $\mathcal{I}$ is at most countable, $f$ is in $\sigma-S(X)$. Conversely, each element of $S(X)$ or $\sigma-S(X)$ may be written in this prescribed form. We include the proof of the next lemma for the sake of completeness [5, II.1.3].

Lemma 3.1. Suppose $f \in L(\mu, X)$ and $\varepsilon>0$. Then there exists $g \in \sigma-S(X)$ such that $\|f-g\|_{\infty}<\varepsilon$.

Proof. Let $A$ be a set of measure zero in $\Omega$ such that $f(\Omega \backslash A)$ is separable in $X$. Then for any $\varepsilon>0$ there is a countable collection $\left\{B\left(x_{i}, \frac{\varepsilon}{2}\right): x_{i} \in X, i=1,2, \ldots\right\}$ of open balls that covers $f(\Omega \backslash A)$. Let the corresponding collection of measurable sets $\left\{U_{i}=f^{-1}\left(B\left(x_{i}, \frac{\varepsilon}{2}\right)\right): i=1,2, \ldots\right\}$ be given in $\Omega \backslash A$. Define a disjoint sequence of measurable sets in $\Omega \backslash A$ as follows: $V_{1}=U_{1}$, and $V_{n+1}=U_{n+1} \backslash \bigcup_{i=1}^{n} U_{i}$ for each $n=1,2, \ldots$ It is clear that $\left\{V_{i}\right\}$ covers $\Omega \backslash A$. Some of the sets in the sequence may be empty, in which case we delete them from the sequence. Let $e_{i} \in L^{\infty}(\mu)$ be the characteristic function of $V_{i}$. Take $t_{i} \in V_{i}$ and let $y_{i}=f\left(t_{i}\right)$. Let $g=\sum e_{i} \vec{y}_{i}$. Then $(f-g) \in L^{\infty}(\mu, X)$ and $\|f-g\|_{\infty}<\varepsilon$.

We have the following consequence of Lemma 3.1.

Lemma 3.2. The subspace $E(X) \cap \sigma-S(X)$ is dense in the Köthe-Bochner space $E(X)$.

Proof. Let $f \in E(X)$ and let $\varepsilon>0$. By Lemma 3.1, there is $g \in \sigma-S(X)$ such that $(g-f) \in L^{\infty}(\mu, X)$ and $\|g-f\|_{\infty}<\varepsilon$. We have $|g|_{X} \leq|g-f|_{X}+|f|_{X}$, and since $L^{\infty}(\mu) \subset E$, it follows that $g \in E(X)$ and $\|g-f\|_{E(X)}<\varepsilon$.

We will make a brief comparison of the spaces defined in the previous section and the Köthe-Bochner spaces under the assumption that $S$ is an infinite set and $X$ is an infinite dimensional Banach space. Note that we have $E=L^{\infty}(\mu)=$ $C(S)=F$. However, when $X$ is infinite dimensional, $C(S, X)$ is a proper closed subspace of $L^{\infty}(\mu, X)$. On the other hand, the opposite is also possible. When $E=L^{1}(\mu)=\operatorname{cl}\left(L^{\infty}(\mu) 1\right)=F$, we have $L^{1}(\mu, X)=L_{\pi}^{1}(\mu, X)$. This follows from the fact that $S(X)$ is dense in $L^{1}(\mu, X)$ and that $M(X)$ is dense in $L_{\pi}^{1}(\mu, X)$ with $S(X) \subset M(X)$. In the case when $F=I(1)$ is a proper ideal of $E$, we have the isometric inclusions

$$
F_{\pi}(X) \subset F(X) \subset E(X) .
$$


The embedding of $F(X)$ into $E(X)$ is always proper. However, if $L^{\infty}(\mu, X) \subset$ $F_{\pi}(X)$ or equivalently if $F$ has order continuous norm, then $F_{\pi}(X)=F(X)$. Otherwise that inclusion is also proper (see Remark 5.3 and Remark 5.5). Note that a Banach lattice $F$ is said to have order continuous norm if $\left\{x_{\alpha}\right\}$ is an increasing net of non-negative elements in $F$ with $\sup x_{\alpha}=x$ implies that $\left\{x_{\alpha}\right\}$ converges to $x$ in norm.

When $S$ is a Stonian compact Hausdorff space, a $C(S)$-module $M$ is called a Kaplansky module [8] if, for each $x \in M$, the set $\{a \in C(S): a x=0\}$ is a band in $C(S)$. Recall that an ideal $I$ in a vector lattice $F$ is called a band if $A \subset I$ and $\sup A$ exists in $F \operatorname{implies} \sup A \in I$. Every Dedekind complete vector lattice is a Kaplansky module over its ideal center [13, II.2.10].

Lemma 3.3. $E(X)$ is a Kaplansky $L^{\infty}(\mu)$-module.

Proof. Suppose $a_{\alpha} \in L_{+}^{\infty}(\mu)$ for all $\alpha \in \mathcal{I}$ and $\sup a_{\alpha}=a \in L^{\infty}(\mu)$. Also suppose, for some $f \in E(X)$, that we have $a_{\alpha} f=0$ for all $\alpha \in \mathcal{I}$. Then $\left\|a_{\alpha} f\right\|_{E(X)}=0$ implies $a_{\alpha}|f|_{X}=0$ for each $\alpha \in \mathcal{I}$ in $E$. Since $E$ is a Kaplansky module, we have $a|f|_{X}=0$. Hence $a f=0$ and $E(X)$ is a Kaplansky module.

The following theorem is an analogue of Theorem 2.3 for Köthe-Bochner spaces. Note that it is weaker than Theorem 2.3, since (iic) requires more of the operator $T$ than (iib).

Theorem 3.4. Let $E(X)$ be the Köthe-Bochner space associated with a Banach function space $E$ on the probability measure space $(\Omega, \Sigma, \mu)$ and with a Banach space $X$. Let $T$ be an operator on $E(X)$. Consider the following conditions:

(i) $T$ is a multiplication operator.

(iia) For each $x \in X$, the cyclic subspace $E(\vec{x})$ is left invariant by $T$.

(iic) $T$ commutes with $L^{\infty}(\mu)$ on $E(X)$.

Then (i) $\Leftrightarrow$ (iia) and (iic).

Proof. We only need to prove (iia) and (iic) $\Rightarrow($ i). Let $F=I(1)$ in $E$. As we noted above, the definitions of the norms show that $F_{\pi}(X)$ is a closed subspace of $E(X)$. Also, for each $x \in X$, we have $E(\vec{x})=\operatorname{cl}\left(L^{\infty}(\mu) \vec{x}\right)=F(\vec{x}) \subset F_{\pi}(X)$. Then condition (iia), in particular, implies that $T(a \vec{x}) \in F_{\pi}(X)$ for all $a \in L^{\infty}(\mu)$ and $x \in X$. That is, $T$ maps $M(X)$, the submodule of $E(X)$ generated by the constant functions, into $F_{\pi}(X)$. Since $T$ is bounded and $M(X)$ is dense in $F_{\pi}(X)$ by construction, when we pass to closure in $T(M(X)) \subset F_{\pi}(X)$, we obtain that $F_{\pi}(X)$ is invariant under $T$. Then (iia), (iic) and Theorem 2.3 imply that $T$ is a multiplication operator on $F_{\pi}(X)$. So there is an $a \in L^{\infty}(\mu)$ such that $T(f)=a f$ for all $f \in F_{\pi}(X)$. Let $g \in E(X) \cap \sigma-S(X)$. Suppose that $\left\{e_{i}\right\}$ is a sequence of disjoint idempotents in $L^{\infty}(\mu)$ and $\left\{x_{i}\right\}$ is a sequence of distinct elements of $X$ such that $g=\sum e_{i} \vec{x}_{i}$. We will assume without loss of generality that $\sup e_{i}=1$. For each positive integer $n$, let $\chi(n)=\sum_{1 \leq i \leq n} e_{i} \in L^{\infty}(\mu)$. Then $\chi(n) g \in S(X) \subset$ $M(X) \subset F_{\pi}(X)$, for each $n$. Hence, by (iic) we have, for each $n$,

$$
\chi(n) T(g)=T(\chi(n) g)=a(\chi(n) g)=\chi(n)(a g) .
$$

Since $\sup \chi(n)=\sup e_{i}=1$ and $E(X)$ is a Kaplansky module, we have $T(g)=a g$ for all $g \in E(X) \cap \sigma-S(X)$. By Lemma 3.2, we have that $E(X) \cap \sigma-S(X)$ is dense in $E(X)$. Therefore $T$ is multiplication by $a \in L^{\infty}(\mu)$ on all of $E(X)$. 
In the next section we will consider the application of Theorem 3.4 to a functional equation that Calabuig, Rodríguez, and Sánchez-Pérez defined in [4] and used as a criteria for identifying multiplication operators on Köthe-Bochner spaces.

\section{The Functional EQUation}

Let $T$ be an operator on the Köthe-Bochner space $E(X)$. In [4] the following functional equation was considered:

$$
T\left(a\left\langle f, x^{*}\right\rangle \vec{x}\right)=a\left\langle T(f), x^{*}\right\rangle \vec{x}
$$

for all $a \in L^{\infty}(\mu), f \in E(X), x \in X$ and $x^{*} \in X^{*}$. Here $\left\langle x, x^{*}\right\rangle$ denotes the action of the Banach dual $X^{*}$ on the Banach space $X$. In the functional equation, however, $\left\langle f, x^{*}\right\rangle \in E$ and

$$
\left\langle f, x^{*}\right\rangle(t)=\left\langle f(t), x^{*}\right\rangle
$$

for all $t \in \Omega \backslash A$ for some set $A$ of measure zero in $\Omega$. The authors show in [4. Corollary 2.3] that when $E$ has order continuous norm, $T$ is a multiplication operator if and only if $T$ satisfies the functional equation. We intend to apply Theorem 2.3 and Theorem 3.4 to extend the result to operators on spaces $F_{\pi}(X)$ and to operators on all Köthe-Bochner spaces $E(X)$.

Initially we need to show that the terms of the equation make sense in $F_{\pi}(X)$. Let $f \in C(K, X)$; then it is clear that $\left\langle f, x^{*}\right\rangle$ makes sense when defined pointwise at each $t \in K$, and we have $\left\langle f, x^{*}\right\rangle \in C(K) \subset F$. For each $t \in K$, we have $\left|\left\langle f(t), x^{*}\right\rangle\right| \leq\|f(t) \mid\|\left\|x^{*}\right\|$. Then in $F$, we have $\left|\left\langle f, x^{*}\right\rangle\right| \leq|f|_{X}\left\|x^{*}\right\|$. Therefore for a fixed $x^{*} \in X^{*}$ and for all $f \in C(K, X) \subset F_{\pi}(X)$, we get $\left\|\left\langle f, x^{*}\right\rangle\right\|_{F} \leq$ $\|f\|_{F(X)}\left\|x^{*}\right\|$. This means that for each $x^{*} \in X^{*}$, the mapping $f \longmapsto\left\langle f, x^{*}\right\rangle$ defines a bounded linear transformation on the subspace $C(K, X)$ of $F_{\pi}(X)$ into $F$. Since $C(K, X)$ is dense in $F_{\pi}(X)$, the map extends uniquely to all of $F_{\pi}(X)$. For each $f \in F_{\pi}(X) \backslash C(K, X)$ let $\left\langle f, x^{*}\right\rangle \in F$ denote its image under the extension. Also for each $x \in X$, by Lemma 2.2 we have $F(\vec{x}) \cong F$. Let $\left\langle f, x^{*}\right\rangle \vec{x} \in F(\vec{x})$ denote the image of $\|x\|\left\langle f, x^{*}\right\rangle \in F$ in this isomorphism. Now it is clear that we can write the functional equation in $F_{\pi}(X)$.

Lemma 4.1. Let $T$ be an operator on $F_{\pi}(X)$. Suppose $T$ satisfies

$$
T\left(a\left\langle f, x^{*}\right\rangle \vec{x}\right)=a\left\langle T(f), x^{*}\right\rangle \vec{x}
$$

for all $a \in C(K), f \in F_{\pi}(X), x \in X$ and $x^{*} \in X^{*}$. Then

(iia) For each $x \in X$, the cyclic subspace $F(\vec{x})$ is left invariant by $T$.

(iib) For each $x \in X, T$ commutes with $C(K)$ on $F(\vec{x})$.

Proof. Let $a \in C(K)$ and $x \in X$. Take $x^{*} \in X^{*}$ such that $\left\langle x, x^{*}\right\rangle=1$. Then

$$
T(a \vec{x})=T\left(a\left\langle\vec{x}, x^{*}\right\rangle \vec{x}\right)=a\left\langle T(\vec{x}), x^{*}\right\rangle \vec{x} .
$$

By the discussion preceding the lemma, since $\left\langle T(\vec{x}), x^{*}\right\rangle \in F$, we have $\left\langle T(\vec{x}), x^{*}\right\rangle$ $\vec{x} \in F(\vec{x})$. Therefore $T(C(K) \vec{x}) \subset F(\vec{x})$. This implies (iia). Let $a, b \in C(K)$ and $x \in X$. Choose $x^{*} \in X^{*}$ such that $\left\langle x, x^{*}\right\rangle=1$. Then

$$
T(a b \vec{x})=T\left(a b\left\langle\vec{x}, x^{*}\right\rangle \vec{x}\right)=a\left(b\left\langle T(\vec{x}), x^{*}\right\rangle \vec{x}\right)=a T\left(b\left\langle\vec{x}, x^{*}\right\rangle \vec{x}\right)=a T(b \vec{x}) .
$$

When we pass to closure, we have (iib).

Now we can state the following corollary to Theorem 2.3 and prove it by using Lemma 4.1. 
Corollary 4.2. Let $T$ be an operator on $F_{\pi}(X)$. Then the following are equivalent:

(i) $T$ is a multiplication operator.

(iii) The equality

$$
T\left(a\left\langle f, x^{*}\right\rangle \vec{x}\right)=a\left\langle T(f), x^{*}\right\rangle \vec{x}
$$

holds for all $a \in C(K), f \in F_{\pi}(X), x \in X$ and $x^{*} \in X^{*}$.

Next we consider the functional equation in the case of Köthe-Bochner spaces. In this case difficulties arise when $1 \in E$ is not a quasi-interior point in $E$. With $F=I(1)$, suppose $\phi \in E \backslash F$. Then, for each $x \in X$, we have $\phi \vec{x} \notin E(\vec{x})=F(\vec{x})$ by Lemma 2.2. So for each $x \in X$, we need to consider the submodule of $E(X)$ that is given by $[\vec{x}]:=\{\phi \vec{x}: \phi \in E\}$. We call $[\vec{x}]$ the band-type submodule generated by $\vec{x} \in E(X)$.

Lemma 4.3. Let $E(X)$ be a Köthe-Bohner space and let $x \in X$.

(1) The band-type submodule $[\vec{x}]$ is isometric and lattice isomorphic to the Banach function space $E$.

(2) Suppose that for some $f \in E(X)$, there is a family of upwards directed idempotents $\left\{e_{\alpha}: \alpha \in \mathcal{I}\right\}$ in $L^{\infty}(\mu)$ such that $\sup e_{\alpha}=1$ and $e_{\alpha} f \in[\vec{x}]$ for each $\alpha \in \mathcal{I}$. Then $f \in[\vec{x}]$.

Proof. Clearly $[\vec{x}]$ is a submodule of $E(X)$ and it is clear that $E \subset L(\mu)$ induces on $[\vec{x}]$ a vector lattice structure with respect to which it is a Dedekind complete vector lattice with weak order unit $\vec{x}$. Let $\phi \in E$. Then

$$
\|\phi \vec{x}\|_{E(X)}=\left\||\phi \vec{x}|_{X}\right\|_{E}=\||\phi|\| x\|\|_{E}=\|\phi\|_{E}\|x\|=\|\| x\|\phi\|_{E} .
$$

Hence the correspondence $\phi \vec{x} \longleftrightarrow\|x\| \phi$ gives the isometric lattice isomorphism between the two spaces. This completes part (1). To prove part (2), assume the conditions in the statement of (2). Then, there is a collection of functions $\left\{\phi_{\alpha}: \alpha \in \mathcal{I}\right\}$ in $E$ such that $e_{\alpha} f=\phi_{\alpha} \vec{x}$ for each $\alpha \in \mathcal{I}$ and $e_{\alpha} \phi_{\beta}=\phi_{\alpha}$ whenever $\alpha \leq \beta$ in the order of the index set $\mathcal{I}$. Then $e_{\alpha}|f|_{X}=\left|\phi_{\alpha}\right|\|x\|$ for each $\alpha \in \mathcal{I}$. It follows that $\|x\|\left(\sup \left|\phi_{\alpha}\right|\right)=|f|_{X}$. Moreover, there exists an idempotent $e \in L^{\infty}(\mu)$ such that for all $\alpha \in \mathcal{I}$, we have $e\left|\phi_{\alpha}\right|=\phi_{\alpha}^{+}$and $(1-e)\left|\phi_{\alpha}\right|=\phi_{\alpha}^{-}$. Let $a=e-(1-e)$. Hence $e_{\alpha} a|f|_{X}=a\left|\phi_{\alpha}\right|\|x\|=\phi_{\alpha}\|x\|$ and $e_{\alpha} f=\phi_{\alpha} \vec{x}=e_{\alpha}\left(\frac{a}{\|x\|}|f|_{X}\right) \vec{x}$ for each $\alpha \in \mathcal{I}$. Since $E(X)$ is a Kaplansky module (Lemma 3.3), we have

$$
f=\frac{a}{\|x\|}|f|_{X} \vec{x} \in[\vec{x}] .
$$

This proves part (2).

Lemma 4.4. Let $T$ be an operator on the Köthe-Bochner space $E(X)$. Suppose $T$ satisfies

$$
T\left(a\left\langle f, x^{*}\right\rangle \vec{x}\right)=a\left\langle T(f), x^{*}\right\rangle \vec{x}
$$

for all $a \in L^{\infty}(\mu), f \in F_{\pi}(X), x \in X$ and $x^{*} \in X^{*}$. Then

(iia) For each $x \in X$, the cyclic subspace $E(\vec{x})$ is left invariant by $T$,

(iic) $T$ commutes with $L^{\infty}(\mu)$ on $E(X)$. 
Proof. We will prove (iic) first. Observe that $a\left\langle f, x^{*}\right\rangle=\left\langle a f, x^{*}\right\rangle$ for all $a \in$ $L^{\infty}(\mu), f \in E(X)$ and $x^{*} \in X^{*}$. Then for each $x \in X$, (iii) implies

$$
T\left(a\left\langle f, x^{*}\right\rangle \vec{x}\right)=a\left\langle T(f), x^{*}\right\rangle \vec{x}=\left\langle a T(f), x^{*}\right\rangle \vec{x}
$$

and

$$
T\left(a\left\langle f, x^{*}\right\rangle \vec{x}\right)=T\left(\left\langle a f, x^{*}\right\rangle \vec{x}\right)=\left\langle T(a f), x^{*}\right\rangle \vec{x}
$$

for all $a \in L^{\infty}(\mu), f \in E(X)$ and $x^{*} \in X^{*}$. Then by Lemma 4.3(1), we have $\left\langle a T(f), x^{*}\right\rangle=\left\langle T(a f), x^{*}\right\rangle$ for all $x^{*} \in X$ when we take some fixed $a \in L^{\infty}(\mu), f \in$ $E(X)$. Since the functions in $E(X)$ have separable range, by an often used result [5, II.2.7], we have $a T(f)=T(a f)$. That is, (iii) implies (iic). Next we will show that for each $x \in X$, the operator $T$ leaves the band-type submodule $[\vec{x}]$ invariant. Let $\phi \in E$. Consider the sequence of measurable sets $V_{n}=\{t:|\phi(t)| \leq n\}, n=1,2, \ldots$. Let $e_{n}$ denote the characteristic function of $V_{n}$. Then the sequence $\left\{e_{n}\right\}$ is increasing and $\sup e_{n}=1$ in $L^{\infty}(\mu)$. We have $e_{n} \phi \in L^{\infty}(\mu)$, for each $n$. Also take $x^{*} \in X^{*}$ such that $\left\langle x, x^{*}\right\rangle=1$. Then, by (iic),

$$
e_{n} T(\phi \vec{x})=T\left(e_{n} \phi \vec{x}\right)=T\left(e_{n} \phi\left\langle\vec{x}, x^{*}\right\rangle \vec{x}\right)=e_{n} \phi\left\langle T(\vec{x}), x^{*}\right\rangle \vec{x}
$$

That is $e_{n} T(\phi \vec{x}) \in[\vec{x}]$, for each $n=1,2, \ldots$ Then Lemma 4.3(2) implies that $T(\phi \vec{x}) \in[\vec{x}]$. That is, $T$ leaves $[\vec{x}]$ invariant. Furthermore (iic) implies $T$ commutes with $L^{\infty}(\mu)$ on $[\vec{x}]$. By Lemma $4.3(1)$, we have $E \cong[\vec{x}]$. Therefore $T$ restricted to $[\vec{x}]$ corresponds to an operator on $E$ that commutes with the ideal center $Z(E)=$ $L^{\infty}(\mu)$. Since $E$ is a Dedekind complete Banach lattice, it is well known that its ideal center is maximal abelian (e.g., [15, Proposition 2.1]). So $T$ is a multiplication operator on $E$ and therefore on $[\vec{x}]$. That is, for some $a_{x} \in L^{\infty}(\mu)$, for all $\phi \in E$, we have $T(\phi \vec{x})=a_{x} \phi \vec{x}$. When $a \in L^{\infty}(\mu) \subset E$, we have $T(a \vec{x})=a_{x} a \vec{x} \in$ $E(\vec{x})$. Therefore $T$ leaves the cyclic subspace $E(\vec{x})$ invariant. That is, (iii) implies (iia).

The use of Lemma 4.4 and Theorem 3.4 yield the following corollary to Theorem 3.4 .

Corollary 4.5. Let $T$ be an operator on the Köthe-Bochner space $E(X)$. Then the following are equivalent:

(i) $T$ is a multiplication operator.

(iii) The equality

$$
T\left(a\left\langle f, x^{*}\right\rangle \vec{x}\right)=a\left\langle T(f), x^{*}\right\rangle \vec{x}
$$

holds for all $a \in L^{\infty}(\mu), f \in E(X), x \in X$ and $x^{*} \in X^{*}$.

\section{EXAMPles, COMPLEMENTARY RESUlts AND REMARKS}

Throughout the section, $E$ will be a Banach function space in $L^{1}(\mu)$ with weak order unit 1 and $F$ will be the Banach function space given by the closed ideal generated by 1 in $E$. We will think of $F_{\pi}(X)$ as the closure of $S(X)$ in the Köthe-Bochner space $E(X)$. For Köthe-Bochner spaces, in general, the conditions of Theorem 2.3 (i.e., (iia) and (iib)) are not sufficient to identify the multiplication operators. Below we give examples of operators on $E(X)$ that satisfy conditions (iia) and (iib) of Theorem 2.3 but are not multiplication operators. 
Example 5.1. Suppose that $F_{\pi}(X)$ is a proper closed subspace of $E(X)$. Let $\mathcal{N}$ denote the non-zero operators on $E(X)$ that are zero on $F_{\pi}(X)$. The set $\mathcal{N}$ is nonempty (via the Hahn-Banach Theorem). Take $T \in \mathcal{N}$ and $a \in L^{\infty}(\mu)$ considered as a multiplication operator on $E(X)$. Consider the operator $T+a$ on $E(X)$. When restricted to $F_{\pi}(X)$ we have that $T+a=a$. So $T+a$ is a multiplication operator on $F_{\pi}(X)$ and leaves it invariant. Hence, by Theorem 2.3, $T+a$ satisfies conditions (iia) and (iib) on $F_{\pi}(X)$. Recall from the proof of Theorem 3.4 that the cyclic subspace $E(\vec{x})=F(\vec{x}) \subset F_{\pi}(X)$ for each $x \in X$. That is, $T+a$ satisfies conditions (iia) and (iib) on $E(X)$ but is not a multiplication operator on $E(X)$. So all operators in the class $\mathcal{N}+L^{\infty}(\mu)$ satisfy conditions (iia) and (iib) on $E(X)$ but are not multiplication operators. When $X$ is infinite dimensional and $S$ is infinite with $E=L^{\infty}(\mu)=C(S)=F$, we have that $F_{\pi}(X)=C(S, X), E(X)=L^{\infty}(\mu, X)$ and $C(S, X)$ is a proper closed subspace of $L^{\infty}(\mu, X)$. Other examples are obtained when $F$ is a proper ideal of $E$ because in all such cases $F_{\pi}(X)$ is a proper closed subspace of $E(X)$. See Remark 5.5 below for even more examples on which this type of operator may be constructed.

Next we verify a statement made in Section 3 on Köthe-Bochner spaces while comparing the spaces $F(X)$ and $F_{\pi}(X)$. Initially we need to prove the following result on Köthe-Bochner spaces.

Theorem 5.2. Let $E$ be a Banach function space and let $X$ be an infinite dimensional Banach space. Then $S(X)$ is dense in $E(X)$ if and only if $E$ has order continuous norm.

Proof. It is well known that if $E$ has order continuous norm, then $S(X)$ is dense in $E(X)$ [4, 10]. To complete the proof we will show that if $E$ does not have order continuous norm, then $S(X)$ is not dense in $E(X)$. If 1 is not a quasi-interior point of $E$, then there is nothing to prove, since in that case the closure of $S(X)$ in $E(X)$ equals $F_{\pi}(X)$ (with $F=I(1)$ ) and $F_{\pi}(X)$ is a proper subspace of $E(X)$. Therefore let 1 be a quasi-interior point of $E$ and suppose $E$ does not have order continuous norm. Then there is an increasing squence $\left\{\chi_{n}\right\}$ of idempotents in $L^{\infty}(\mu)$ such that $\sup \chi_{n}=1$ but $\left\{\chi_{n}\right\}$ does not converge to 1 in norm in $E$ (e.g., [13, II.5.10 or V.3.6]). This means that $\left\{\chi_{n}\right\}$ does not converge in $E$ because the only limit it can have is 1 . So $\left\{\chi_{n}\right\}$ is not a Cauchy sequence. Then for some $\delta>0$ we can choose a subsequence $\left\{\chi_{k_{n}}\right\}$ such that $\delta \leq\left\|\chi_{k_{n}}-\chi_{k_{m}}\right\|_{E}$ when $n \neq m$ and $\sup \chi_{k_{n}}=1$.

Define a disjoint sequence of idempotents by setting $e_{1}=\chi_{k_{2}}$ and $e_{i}=\chi_{k_{i+1}}-\chi_{k_{i}}$ for all $i \geq 2$. Clearly $\delta \leq\left\|e_{i}\right\|_{E}$ for all $i$ and $\sup \sum_{i=1}^{n} e_{i}=1$. Since $X$ is infinite dimensional we can choose a distinct sequence $\left\{x_{i}\right\}$ on the unit sphere of $X$ such that $\left\|x_{i}-x_{j}\right\|>1 / 2$ when $i \neq j$. Now define a function $f \in L^{\infty}(\mu, X)$ by setting $f=\sum e_{i} \vec{x}_{i}$. Clearly $f \in E(X)$, and we will show that $f$ is not in the closure of $S(X)$.

Given any $\varepsilon>0$, suppose there is $g \in S(X)$ such that $\|g-f\|_{E(X)}<\varepsilon \delta$. There is a finite distinct subset $\left\{y_{k}: 1 \leq k \leq n\right\}$ in $X$ and a disjoint set of idempotents $\left\{\gamma_{k}: 1 \leq k \leq n\right\}$ in $L^{\infty}(\mu)$ with $\sum \gamma_{k}=1$ such that $g=\sum \gamma_{k} \vec{y}_{k}$. For each $i$, we have

$$
e_{i}(g-f)=e_{i}\left(g-\vec{x}_{i}\right)=e_{i}\left(\sum_{k=1}^{n} \gamma_{k}\left(\vec{y}_{k}-\vec{x}_{i}\right)\right)
$$


Then

$\min _{1 \leq k \leq n}\left\|y_{k}-x_{i}\right\| \delta \leq \min _{1 \leq k \leq n}\left\|y_{k}-x_{i}\right\|\left\|e_{i}\right\|_{E} \leq\left\|e_{i}(g-f)\right\|_{E(X)} \leq\|g-f\|_{E(X)}<\varepsilon \delta$.

Therefore for each $i$ there exists some $y_{k}, 1 \leq k \leq n$, such that $\left\|y_{k}-x_{i}\right\|<\varepsilon$. But there are only finitely many $y_{k}$; therefore, for some $y_{k}$, there must be a pair of indices $i \neq j$ such that both $\left\|y_{k}-x_{i}\right\|<\varepsilon$ and $\left\|y_{k}-x_{j}\right\|<\varepsilon$ hold. This implies that $\left\|x_{i}-x_{j}\right\|<2 \varepsilon$. When $\varepsilon \leq 1 / 4$, this contradicts our initial choice of the sequence $\left\{x_{i}\right\}$. Hence $f$ is not in the closure of $S(X)$ and the proof is complete.

Remark 5.3. It is clear from Theorem 5.2 that when $X$ is infinite dimensional, $F_{\pi}(X)=F(X)$ if and only if $F$ has order continuous norm. The theorem also shows that the hypothesis of the main result of [4, Theorem 1.4] and that of its corollary [4, Corollary 2.3] are equivalent.

To verify a second statement made in Section 3 on Köthe-Bochner spaces while comparing the spaces $F(X)$ and $F_{\pi}(X)$, we need to prove the following result.

Theorem 5.4. Let $E$ be a Banach function space and let $X$ be a Banach space. Suppose 1 is a quasi-interior point of $E$; then $L^{\infty}(\mu, X)$ is dense in $E(X)$.

Proof. Let $g \in E(X) \cap \sigma-S(X)$. Suppose $g=\sum e_{i} \vec{x}_{i}$, where $\left\{e_{i}\right\}$ is a sequence of disjoint idempotents in $L^{\infty}(\mu)$ and $\left\{x_{i}\right\}$ is a sequence of distinct elements in $X$. Let $\mathcal{I}(n)=\left\{i:\left\|x_{i}\right\| \leq n\right\}$ and let the sequence $\left\{g_{n}\right\}$ in $L^{\infty}(\mu, X)$ be defined by

$$
g_{n}=\sum_{i \in \mathcal{I}(n)} e_{i} \overrightarrow{x_{i}}+\sum_{i \notin \mathcal{I}(n)} e_{i}\left(\frac{n}{\left\|x_{i}\right\|} \overrightarrow{x_{i}}\right)
$$

for each $n=1,2, \ldots$ Note that

$$
\begin{aligned}
\left|g_{n}\right|_{X} & =|g|_{X} \wedge n 1 \quad \text { and } \\
\left|g-g_{n}\right|_{X} & =|g|_{X}-\left|g_{n}\right|_{X} \geq 0
\end{aligned}
$$

for each $n=1,2, \ldots$ Since 1 is a quasi-interior point in $E,\left\{|g|_{X} \wedge n 1\right\}$ converges to $|g|_{X}$ in $E$ [13, II.6.3]. Then

$$
\left\|g-g_{n}\right\|_{E(X)}=\left\|\left|g-g_{n}\right|_{X}\right\|_{E}=\left\||g|_{X}-\left|g_{n}\right|_{X}\right\|_{E}
$$

implies that $\left\{g_{n}\right\}$ converges to $g$ in $E(X)$. Hence, by Lemma $3.2, L^{\infty}(\mu, X)$ is dense in $E(X)$.

Remark 5.5. Let $E$ be a Banach function space and let $F=I(1)$. Then, since 1 is a quasi-interior point of $F$, by Theorem 5.4, we have $F_{\pi}(X)=F(X)$ if and only if $L^{\infty}(\mu, X) \subset F_{\pi}(X)$. Note that in such a case, if $X$ is infinite dimensional, then Theorem 5.2 implies that $F$ has order continuous norm. Theorem 5.4 has certain simplifying implications when one looks at examples. Namely, suppose $\phi \in E$ such that $\|\phi\|_{E}=1$ and for some $\varepsilon>0, \varepsilon \leq \phi$. Let $G=I(\phi)$ in $E$. Since $G$ is a Banach function space and $\phi$ is a quasi-interior point in $G$, we may consider the spaces $G_{\pi}(X)$ and $G(X)$. However to do this we need to change the functional representation of $G$ (so that we have $\phi$ corresponding to 1 ). On the other hand, by Lemma 2.2 , one can see that for each $x \in X$, the cyclic subspace $G(\vec{x})$ in $G(X)$ may be identified with the cyclic subspace $E(\phi \vec{x})$ in $E(X)$. In fact, they 
are both lattice isometric to $G$. Then it follows that $G_{\pi}(X) \cong \operatorname{cl}(\phi C(S, X))$ in $E(X)$. Similarly if $f \in L^{\infty}(\mu, X)$, then the definitions of the norms immediately give $\|f\|_{G(X)}=\|\phi f\|_{E(X)}$. Since Theorem 5.4 gives that $G(X)$ is the closure of $L^{\infty}(\mu, X)$ in the $G(X)$-norm, we have $G(X) \cong c l\left(\phi L^{\infty}(\mu, X)\right)$ in $E(X)$. So we may realize the spaces $G_{\pi}(X), G(X)$ as submodules of $E(X)$ without the need to change the functional representation of $G$. In particular let $E$ be an Orlicz space whose Orlicz function does not satisfy the $\Delta_{2}$-condition or let $E$ be a Marcinkiewicz space. Then, it is well known 9 that $E$ does not have order continuous norm and that $F=I(1)$ has order continuous norm. However, since $E$ does not have order continuous norm, there must be $\phi \in E$ such that $G=I(\phi)$ does not have order continuous norm. We may suppose without loss of generality that $\|\phi\|_{E}=1$ and for some $\varepsilon>0, \varepsilon \leq \phi$. Then the above discussion implies that $G_{\pi}(X)$ is a proper subspace of $G(X)$, and this provides yet another collection of Köthe-Bochner spaces on which the operators of Example 5.1 may be constructed.

In conclusion, we return to the discussion in the introduction and compare Theorems 2.3 and 3.4 with the general result about multiplication operators on Banach $C(K)$-modules ([2, Theorem 6.2], 6, Theorem 7]).

Remark 5.6. Let $M$ be a Banach $C(K)$-module and suppose that the set of multiplication operators (i.e., multiplication by an element of $C(K))$ is closed in the weak-operator topology in $\mathcal{L}(M)$, the set of (bounded) operators on $M$. Then we know that $T \in \mathcal{L}(M)$ is a multiplication operator if and only if $T$ leaves invariant each cyclic subspace $M(x)$ for every $x \in M$. In the case of Köthe-Bochner spaces as Banach $L^{\infty}(\mu)$-modules we saw that it is sufficient for $T$ to satisfy the functional equation in order to be a multiplication operator (Corollary 4.5). We also saw in Theorems 2.3 and 3.4 that the conceptual basis of the functional equation requires the operator to leave invariant a very restricted class of cyclic subspaces, namely those given by the constant functions. This is compensated by the commutativity conditions (iib) or (iic). However, that the class of multiplication operators should be weak-operator closed in $\mathcal{L}(E(X))$ or respectively in $\mathcal{L}\left(F_{\pi}(X)\right)$ is not mentioned because the constructions of the spaces $E(X)$ and $F_{\pi}(X)$ guarantee that the set of multiplication operators would be weak-operator closed in either case.

If one takes subalgebras of $L^{\infty}(\mu)$, then the situation changes. Let $E=L^{1}[0,1]$ with Lebesgue measure on $[0,1]$. Take the subalgebra $C[0,1]$ of $L^{\infty}[0,1]$, the center of $E$. Consider an operator $T$ on $E(X)=L^{1}([0,1], X)$ such that, for each $x \in X, T$ leaves $E(\vec{x})$ invariant and commutes with $C[0,1]$ on $E(\vec{x})$. Then $T \in L^{\infty}[0,1]$ (and not in $C[0,1]$ in general). This works because $E$ has order continuous norm. Now let $L^{\infty}[0,1]=C(S)$, where $S$ is hyperstonian, and consider $C(S, X)$. Suppose $T$ is an operator on $C(S, X)$ that leaves the cyclic subspaces of $C(S, X)$ generated by constant functions invariant and commutes with $C[0,1]$ on these cyclic subspaces. Then $T$ need not be a multiplication operator. Initially note that Lemma 2.2 implies that the cyclic subspaces of $C(S, X)$ generated by the constant functions are identified with $C(S)=L^{\infty}[0,1]$. Then note that a remarkable and deep result of de Pagter and Ricker [12] states that the bicommutant of the multiplication operators given by $C[0,1]$ in $\mathcal{L}\left(L^{\infty}[0,1]\right)$ consists of the bounded Riemann integrable functions on $[0,1]$, so that an operator $A$ in $\mathcal{L}\left(L^{\infty}[0,1]\right)$ that commutes with $C[0,1]$ is not in general a multiplication operator in $L^{\infty}[0,1]$. However, using the methods in the proof of Theorem 2.3, one can show that there is a single such operator $A_{T}$ 
such that on $M(X)$ one has that

$$
T\left(\sum a_{i} \vec{x}_{i}\right)=\sum A_{T}\left(a_{i}\right) \vec{x}_{i}
$$

for all $a_{i} \in L^{\infty}[0,1]$ and $x_{i} \in X$ with $i=1,2, \ldots, n$ for any $n$. Then the operator is extended to all of $C(S, X)$ by density and continuity.

\section{REFERENCES}

1. Abramovich, Y. A., Aliprantis, C. D., An invitation to operator theory, Graduate Studies in Mathematics, 50. American Mathematical Society, Providence, RI, 2002. MR1921782 (2003h:47072)

2. Abramovich, Y. A., Arenson, E. L. and Kitover, A. K., Banach $C(K)$-modules and operators preserving disjointness. Pitman Research Notes in Mathematical Series 277, Longman Scientific \& Technical, 1992. MR 1202880 (94d:47027)

3. Abramovich, Y. A., Veksler, A. I. and Koldunov, A. V., Operators preserving disjointness. Dokl. Akad. Nauk USSR 248 (1979), 1033-1036. MR553919 (81e:47034)

4. Calabuig, J.M., Rodríguez, J., Sánchez-Pérez, E.A., Multiplication operators in Köthe Bochner spaces. Journal of Mathematical Analysis and Applications 373 (1)(2011), 316-321. MR2684482(2011k:46057)

5. Diestel, J., Uhl, J. J., Jr., Vector measures. Mathematical Surveys, No. 15. American Mathematical Society, Providence, RI, 1977. MR0453964 (56:12216)

6. Hadwin, D., Orhon, M., Reflexivity and approximate reflexivity for Boolean algebras of projections. J. Func. Anal. 87 (1989), 348-358. MR1026857 (91e:47047)

7. Kaijser S., Some representations of Banach lattices. Ark. Mat. 16 (1978), 179-193. MR.524747 (80f:46020)

8. Kaplansky, I., Modules over operator algebras. American Journal of Mathematics 75 (4) (1953), 839-853. MR0058137 (15:327f)

9. Krasnosel'skii, M.A., Rutickii, Ya.B., Convex functions and Orlicz spaces. P. Noordhoff, Ltd., Groningen, 1961. MR0126722 (23:A4016)

10. Lin, P.-K., Köthe-Bochner function spaces, Birkhäuser Boston, Inc., Boston, MA, 2004. MR2018062 (2005k:46084)

11. Orhon, M., The ideal center of the dual of a Banach lattice. Positivity 14(4) (2010), 841-847. MR2741337(2012d:46054)

12. de Pagter, B., Ricker, W. J., Bicommutants of algebras of multiplication operators. Proc. London Math. Soc. (3) 72(2) (1996), 458-480. MR1367086 (97a:47068)

13. Schaefer, H.H., Banach lattices and positive operators. Springer Verlag, Berlin, Heidelberg, New York, 1974. MR0423039 (54:11023)

14. Veksler, A. I., Cyclic Banach spaces and Banach lattices. Soviet Math. Dokl. 14 (1973), 17741779. MR0361718(50:14163)

15. Wickstead, A. W., Banach lattices with topologically full centre. Vladikavkaz. Mat. Zh. 11 (2009), no. 2, 50-60. MR2529410 (2010h:46024)

Department of Mathematics, Faculty of Science, Istanbul University, VeznecilerISTANBUL, 34134, TURKEY

E-mail address: hduru@istanbul.edu.tr

Department of Mathematics, Community College of Philadelphia, 1700 Spring GarDen Street, Philadelphia, Pennsylvania 19130

E-mail address: akitover@ccp.edu

Department of Mathematics and Statistics, University of New Hampshire, Durham, New HampshiRe 03824

E-mail address: mo@unh.edu 